\title{
Effect of Electron Irradiation on Superconductivity in Single Crystals of $\mathrm{Ba}\left(\mathrm{Fe}_{1-x} \mathrm{Ru}_{x}\right)_{2} \mathrm{As}_{2}(x=0.24)$
}

\author{
R. Prozorov, ${ }^{1,2,}$ M. Kończykowski, ${ }^{3}$ M. A. Tanatar, ${ }^{1,2}$ A. Thaler, ${ }^{1,2}$ S. L. Bud'ko, ${ }^{1,2}$ P. C. Canfield, ${ }^{1,2}$ \\ V. Mishra, ${ }^{4}$ and P. J. Hirschfeld ${ }^{5}$ \\ ${ }^{1}$ The Ames Laboratory, Ames, Iowa 50011, USA \\ ${ }^{2}$ Department of Physics and Astronomy, Iowa State University, Ames, Iowa 50011, USA \\ ${ }^{3}$ Laboratoire des Solides Irradiés, CNRS-UMR 7642 \\ and CEA-DSM-IRAMIS, Ecole Polytechnique, F 91128 Palaiseau cedex, France \\ ${ }^{4}$ Materials Science Division, Argonne National Laboratory, Lemont, Illinois 60439, USA \\ ${ }^{5}$ Department of Physics, University of Florida, Gainesville, Florida 32611, USA
}

(Received 13 May 2014; published 18 November 2014)

\begin{abstract}
A single crystal of isovalently substituted $\mathrm{Ba}\left(\mathrm{Fe}_{1-x} \mathrm{Ru}_{x}\right){ }_{2} \mathrm{As}_{2}(x=0.24)$ is sequentially irradiated with 2.5 MeV electrons up to a maximum dose of $2.1 \times 10^{19} e^{-} / \mathrm{cm}^{2}$. The electrical resistivity is measured in situ at $T=22 \mathrm{~K}$ during the irradiation and ex situ as a function of temperature between subsequent irradiation runs. Upon irradiation, the superconducting transition temperature $T_{c}$ decreases and the residual resistivity $\rho_{0}$ increases. We find that electron irradiation leads to the fastest suppression of $T_{c}$ compared to other types of artificially introduced disorder, probably due to the strong short-range potential of the pointlike irradiation defects. A more detailed analysis within a multiband scenario with variable scattering potential strength shows that the observed $T_{c}$ versus $\rho_{0}$ is fully compatible with $s_{ \pm}$pairing, in contrast to earlier claims that this model leads to a too rapid suppression of $T_{c}$ with scattering.
\end{abstract}

DOI: 10.1103/PhysRevX.4.041032

There are several experimental approaches to study the energy gap structure in superconductors. One of them is to measure the change of the superconducting transition temperature $T_{c}$ with artificially introduced disorder. Since impurity scattering mixes the superconducting order parameter at different points on the Fermi surface, controlled potential disorder may be considered a phase-sensitive probe of gap symmetry. It is well known that while the gap and critical temperature of an isotropic $s$-wave superconductor are insensitive to nonmagnetic disorder [1,2], superconducting states with different gap symmetries and structures may be extremely sensitive [3-9]. In the case of iron-based superconductors, the predictions for the effect of disorder differ for various possible pairing states and depend on details of the model. In particular, models involving repulsive interactions, including popular spin fluctuation models (for a review, see Ref. [10]). predict states where the order parameter changes signs between sheets of the Fermi surface, generically called $s_{ \pm}$here, whereas models involving

\footnotetext{
*Corresponding author. prozorov@ameslab.gov

Published by the American Physical Society under the terms of the Creative Commons Attribution 3.0 License. Further distribution of this work must maintain attribution to the author(s) and the published article's title, journal citation, and DOI.
}

Subject Areas: Condensed Matter Physics,

Strongly Correlated Materials,

Superconductivity

orbital fluctuations $[5,6]$ and attractive interactions predict no sign change $\left(s_{++}\right)$. The effect of disorder has also been studied in the coexisting superconducting and long-range magnetic order phase [8]. These different approaches can be studied within a phenomenological multiband theory that for some parameters predicts a crossover from the $s_{ \pm}$to the $s_{++}$state [7].

The major experimental problem in the studies of the effect of disorder is the actual introduction of pointlike defects with a minimal impact on the material itself. In the case of chemical substitutions, there is always a question of whether the foreign ions change not only the scattering but other parameters, such as chemical potential and the band structure. These studies revealed "slow" $T_{c}$ suppression in 122 systems, which was interpreted as the evidence for $s_{++}$ pairing [11]. Recently, Wang et al. criticized this conclusion by extending the phenomenological multiband impurity scattering model to include gap anisotropy and by exploring the effect of differing ratios of intra- to interband scattering matrix elements [9]. They showed that the rate of $T_{c}$ suppression depended sensitively on this ratio and argued that the relatively slow suppression of $T_{c}$ in some chemically doped Fe-based materials was due to dominant intraband scattering. Indeed, it is clear that different ions embedded in different hosts can result in very different scattering strengths $[12,13]$. With this perspective, it is not so surprising that different studies even for the same 
impurity ion, for example, $\mathrm{Zn}$, show completely different results $[11,12,14]$.

Irradiation with energetic particles is an alternative way to introduce defects. However, the nature of the defects produced depends on the type of irradiation [15]. Heavyion irradiation produces columnar tracks or sausagelike linear defects [16], which are difficult to analyze within simplified pointlike potential scattering models. Yet, the experiments with heavy-ion irradiation in iron pnictides have shown a definitive violation of the Anderson theorem [16-18], and independent measurements of the London penetration depth and $T_{c}$ in $\mathrm{BaCo} 122$ and $\mathrm{BaNi122}$ allowed the elimination of the unknown scattering rate, with the analysis then favoring the $s_{ \pm}$pairing scenario [17-19]. Proton [20-22] and $\alpha$ - particle [23] irradiations were also used to study iron pnictides. Proton irradiation has thus far produced the greatest $T_{c}$ suppression rate [22], albeit 2-7 times slower than reported here for electron irradiation. Analysis of the energy transfer from an accelerated particle smashing into the crystal lattice shows that only electrons with energies of $1-10 \mathrm{MeV}$ produce pointlike defects in the form of interstitial ions and vacancies (Frenkel pairs) that presumably form perfect scattering centers [15]. Indeed, these defects are charged, but the overall charge change is compensated, so that there is a negligible shift of the chemical potential due to irradiation. Protons, $\alpha$ particles, and neutrons most likely produce cascades of clusters of defects, and heavy ions produce columnar tracks. A more detailed and systematic investigation of the connection between the type of the scattering centers (their spatial extent and scattering strength) and pair breaking is needed. The effect of the finite size of the defects on $T_{c}$ suppression rate was studied theoretically in Ref. [24]. The effect of electron irradiation on electrondoped BaCo122 and BaNi122 was compared with the effect on $\mathrm{Ba}(\mathrm{AsP}) 122$ in Ref. [25], and it appears that isovalent systems are closer to the clean limit than chargedoped ones.

Isovalent to iron, ruthenium substitution suppresses long-range magnetic order and induces superconductivity but does not change the compensation condition between holes and electrons [26-30]. Angle-resolved photoemission spectroscopy (ARPES) measurements report no change in the shape of the Fermi surface and Fermi velocities up to $x=0.55$ [29] while other ARPES studies find a crossover from two- to three-dimensional geometry of some holelike pockets of the Fermi surface accompanied by the mass increase for the larger doping levels $x$ [30]. These results were theoretically analyzed in a recent $a b$ initio study [31]. For comparison, in another isovalently substituted system, $\mathrm{BaFe}_{2}\left(\mathrm{As}_{1-x} \mathrm{P}_{x}\right)_{2}$, Fermi surface shrinks with $x$ decreasing from $x=1$ [28] and the effective mass diverges on the approach of the optimal doping at $x=0.3$, consistent with the quantum critical point beneath the dome [32]. For the purpose of this work, it is important that for the doping level discussed here, $x=0.24$, the Ru substitution causes no carrier imbalance.

The discussion of the effect of the disorder in iron pnictides crucially depends on the multiband nature of superconductivity that supports both $s_{ \pm}$and $s_{++}$pairing states [7]. It is, therefore, important to compare the effects of irradiation in iron pnictides with an established two-gap $s_{++}$superconductor, $\mathrm{MgB}_{2}$. However, this is not a simple task, because when two gaps are of different magnitude, even a $s_{++}$superconductor may have a large rate of $T_{c}$ suppression with nonmagnetic scattering depending on the interband coupling [33]. A review of the radiation damage of $\mathrm{MgB}_{2}$ films and polycrystalline samples describes a significant effect of $\alpha$-particle and neutron irradiation on $T_{c}$ and $H_{c 2}$ [34]. Neutron irradiation of $\mathrm{MgB}_{2}$ has led to a complete suppression of $T_{c}$ [35,36], which prompts the question of the nature of defects produced by neutron irradiation with a possibility of a nuclear transmutation of boron. This and other issues, such as the influence of irradiation on intergrain coupling, are also discussed in Ref. [34]. As far as electron irradiation is concerned, both low-dose [37] and higher-dose [38] (comparable to this study) electron irradiations found virtually no change of $T_{c}$ in $\mathrm{MgB}_{2}$ crystals. On the other hand, the irradiation had a dramatic effect on vortex properties converting a Bragg glass to a disordered vortex lattice, increasing $H_{c 2}$ at higher doses of irradiation and enhancing vortex pinning, all of which means that electrons do induce defects in the crystal structure [38]. The connection between elevated vortex pinning, increase of resistivity, and substantial suppression of $T_{c}$ upon electron irradiation was studied in iron pnictides in Ref. [25]. Perhaps the absence of the change in $T_{c}$ after electron irradiation in $\mathrm{MgB}_{2}$ can be understood considering a relatively weak interband coupling in $\mathrm{MgB}_{2}$, where $T_{c}$ is not expected to change much [33]. Neutrons and $\alpha$-particle irradiation most likely create nonpointlike defects and also affect other properties resulting in the $T_{c}$ suppression. Further experiments with larger crystals and the possibility of directly measuring resistivity before and after the irradiation are needed for better comparison between $s_{++} \mathrm{MgB}_{2}$ and possible $s_{ \pm}$iron pnictides.

In this paper, we report in situ and ex situ measurements of the electrical resistivity in a single crystal of isovalently substituted $\mathrm{Ba}\left(\mathrm{Fe}_{1-x} \mathrm{Ru}_{x}\right)_{2} \mathrm{As}_{2} \quad(x=0.24)$ subsequently irradiated with $2.5 \mathrm{MeV}$ electrons of different doses up to $2.1 \times 10^{19} e^{-} / \mathrm{cm}^{2}$. To avoid ambiguity in determination of the scattering rate, we exhibit the change of $T_{c}$ versus measured residual resistivity $\rho_{0}$ and we also calculate the conventional single scattering rate $g^{\lambda}$ to compare our results with previous studies and theoretical predictions. Our results indicate that in this system $T_{c}$ is suppressed very rapidly by pointlike potential scattering. Since this is not possible in $s_{++}$superconductors, our results provide strong evidence for the $s_{ \pm}$pairing mechanism in iron-based systems. 
Samples and irradiation technique.-Single crystals of $\mathrm{Ba}\left(\mathrm{Fe}_{1-x} \mathrm{Ru}_{x}\right)_{2} \mathrm{As}_{2}\left(x=0.24, T_{c 0}=17.8 \mathrm{~K}\right)$ are grown out of self-flux as described in detail in Ref. [27]. The samples are characterized with $\mathrm{x}$-ray diffraction, magnetization, transport, and magneto-optical measurements. The composition is determined by using wavelength dispersive x-ray spectroscopy in a JEOL JXA-8200 electron microprobe. The $2.5 \mathrm{MeV}$ electron irradiation is performed at the SIRIUS Pelletron linear accelerator operated by the Laboratoire des Solides Irradiés (LSI) at the Ecole Polytechnique in Palaiseau, France. The sample with four soldered contacts [39] is mounted inside the cell with a flow of liquid hydrogen at $T \approx 22 \mathrm{~K}$. The in situ electric transport measurements are performed while irradiating the sample with the electron beam of 5-8 $\mu \mathrm{A}$ total current through a $5 \mathrm{~mm}$ diameter diaphragm. This current (which eventually provides the calibration of the irradiation dose) is measured with the Faraday cage placed behind the sample stage. The irradiation rate is about $3 \times 10^{-5} \mathrm{C} / \mathrm{s} \mathrm{cm}^{2}$ and the experiments last several days. For convenience, we express the irradiation dose in $\mathrm{C} / \mathrm{cm}^{2}$. The actual dose in the number of electrons per $\mathrm{cm}^{2}$ can be obtained by dividing this number by the electron charge $\bar{e}$.

Results. - Figure 1(a) shows in situ measurements of four-probe resistivity at $22 \mathrm{~K}$ in BaRu122 single crystal during electron irradiation. The irradiation is stopped twice, the sample warmed up to room temperature, transferred to another cryostat in which $\rho(T)$ is measured, and returned to the irradiation chamber. The contacts are never altered in the process. Warming up to room temperature results in a partial annealing of the induced defects. By analyzing the experiments with different samples and doses, a conservative estimate of the annealing rate is about $30 \%$ or less, after which the defects find a metastable configuration and remain localized. This is verified by remeasuring the same samples after months of storage at room temperature in a desiccator. Figure 1(b) shows temperature-dependent resistivity $\rho(T)$ measured between the irradiation runs in a separate cryostat. Clearly, the resistivity increases and the transition temperature decreases with irradiation. In Fig. 1(c), we show further that to a good approximation, the resistivity increase $\Delta \rho_{0}$ is practically independent of temperature.

Figure 2(a) shows the increase of four-probe in-plane resistivity at $T_{c}$ and extrapolated to $T=0$ [see Fig. 1(b)] as a function of the irradiation dose, and Fig. 2(b) shows the suppression of $T_{c}$ with the electron irradiation dose. Two additional points are from two other samples of BaRu122 with quite different initial $T_{c 0}$ and irradiated at the indicated doses. Apparently they fall onto a universal curve indicating that the rate of suppression of $T_{c}$ is independent of the composition in this material, as expected for the isovalent substitution. If the irradiation had another effect in addition to the introduction of disorder (e.g., shift of the chemical potential), it is unlikely that such universality would be
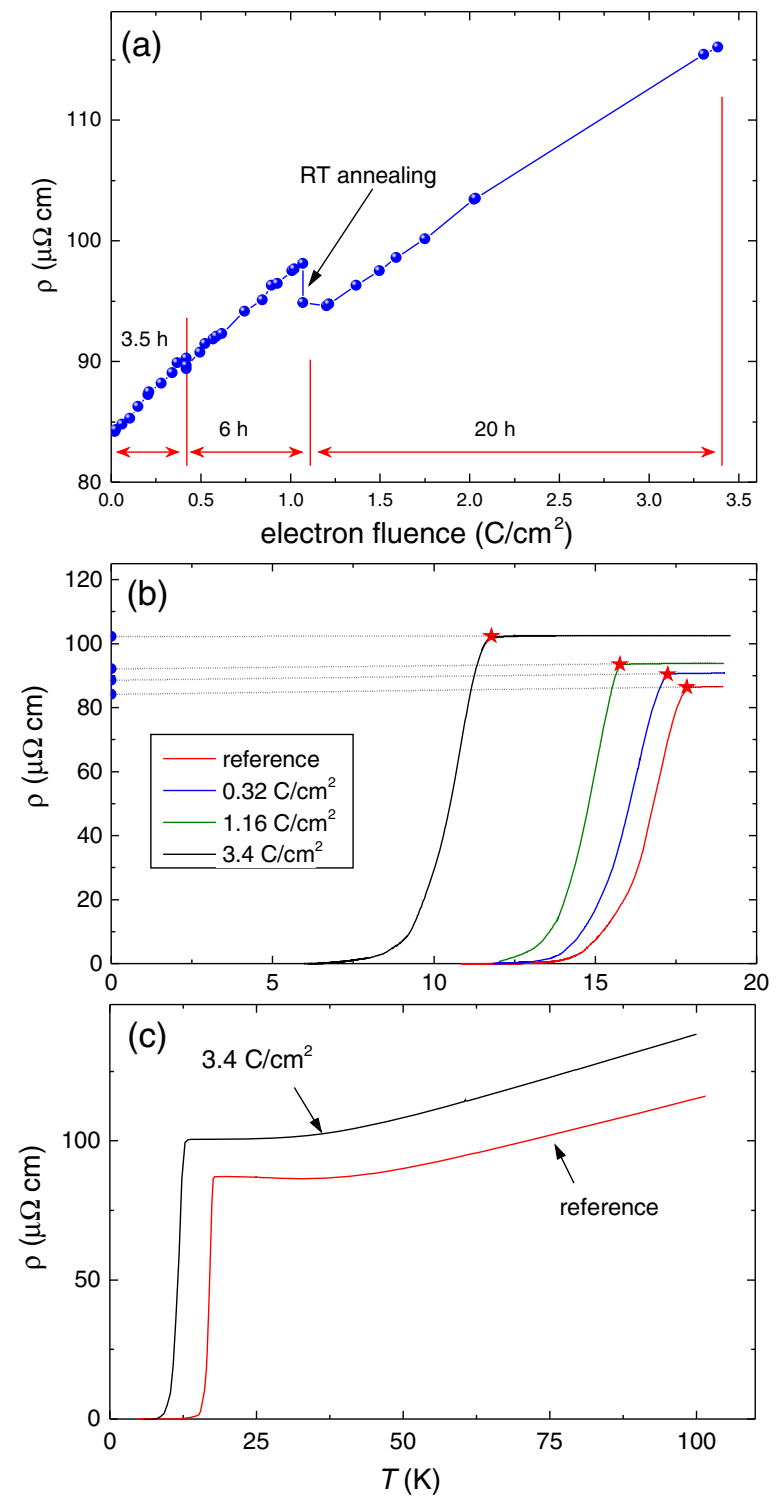

FIG. 1. (a) The in situ measurements of resistivity in $\mathrm{Ba}\left(\mathrm{Fe}_{1-x} \mathrm{Ru}_{x}\right)_{2} \mathrm{As}_{2}(x=0.24)$ at $T \approx 22 \mathrm{~K}$ as a function of the irradiation dose. The breaks in the curve correspond to the extraction of the sample and warming it up to room temperature (RT) resulting in a partial annealing of the defects. (b) The ex situ measurements of resistivity versus temperature between the irradiation runs. Dashed lines show linear extrapolation of $\rho(T)$ from above $T_{c}$ to $T=0$. The estimated residual resistivity is shown by filled circles at $T=0$. (c) Extended temperature range resistivity showing practically uniform shift of the entire curve upon irradiation.

observed. This is also confirmed by the direct Hall effect measurements in which we find practically no change of the Hall coefficient.

Figure 3 compares our results with previous studies of the effect of artificial disorder. Proton irradiation was used on FeCo122 crystals of three different doping levels [21], and $\alpha$-particle were used to irradiate a very thin $\mathrm{Nd} 1111$ 

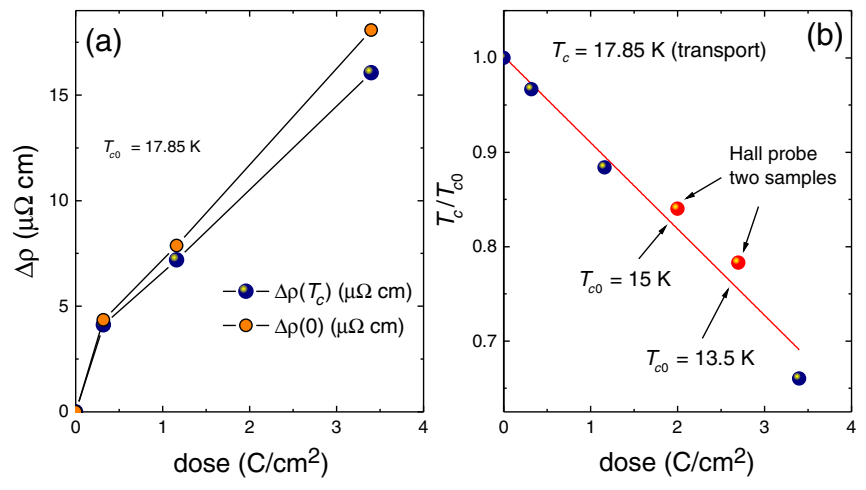

FIG. 2. (a) Change of resistivity of $\mathrm{Ba}\left(\mathrm{Fe}_{1-x} \mathrm{Ru}_{x}\right)_{2} \mathrm{As}_{2}$ ( $x=0.24$ ) at $T_{c}$ and extrapolated to $T=0$ [see Fig. 1(b)] as a function of the irradiation dose. (b) Suppression of $T_{c}$ with the dose of electron irradiation.

crystal [23]. The difference in the rate of $T_{c}$ suppression is consistent with the energy-loss calculations predicting that $\alpha$ particles produce more correlated clusters, protons still produce clusters, and the electrons produce pointlike defects, which are the most efficient pair-breaking scattering centers due to the localized nature of the scattering potential. The analysis presented in these studies, however, used a single dimensionless scattering rate $g$ and was based on the original prediction of the $s_{ \pm}$model $[6,40]$. The authors concluded that the rate of change in $T_{c}(g)$ is too slow for the $s_{ \pm}$scenario. In our view, this conclusion has several flaws. First is the assumption of a single scattering rate $g$ for a single type of defects, although these types of irradiation tend to create a distribution of defects. Secondly,

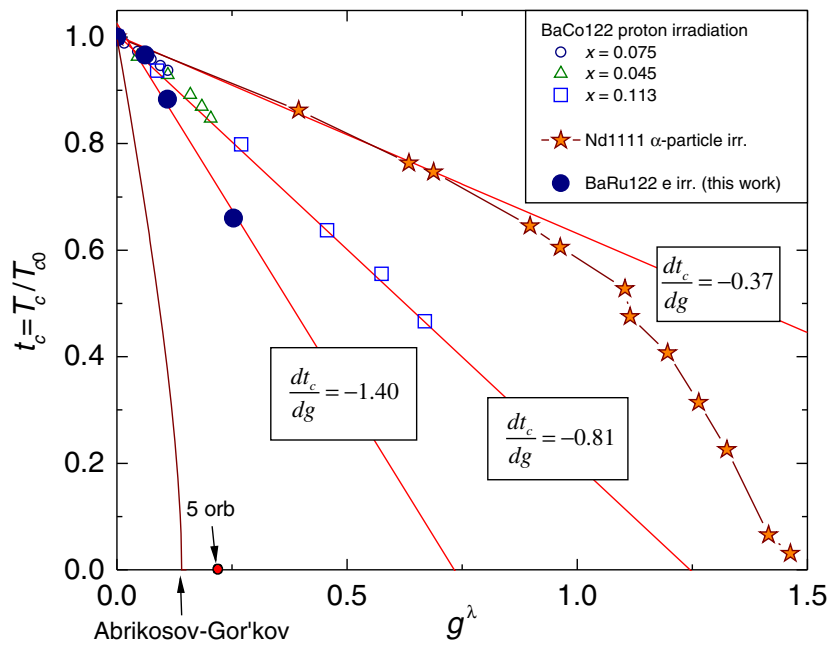

FIG. 3. Comparison of the $T_{c}$ suppression by three irradiation techniques used to introduce artificial disorder in iron pnictides. The single effective dimensionless scattering rate $g^{\lambda}$ is calculated from the penetration depth and resistivity; see text for description. Abrikosov-Gor'kov theory for an isotropic $s$-wave superconductor with magnetic impurities (solid line) and a critical scattering rate within a five-band $s_{ \pm}$model [6] are also shown. in these works, $g$ is estimated within an isotropic Fermi gas model where only mass is renormalized and assumed to represent only interband scattering. The third problem is the treatment of the scattering in the $s_{ \pm}$scenario, where equal densities of states and equal gap magnitudes on two bands are assumed (we refer to this as the symmetric model). This set of assumptions indeed produces $T_{c}$ suppression at a rate identical to the Abrikosov-Gor'kov rate [2], but relaxing any of them produces a much slower relaxation rate in an $s_{ \pm}$state [9].

In the simplest form, the dimensionless scattering rate can be defined using the Drude model as [41]

$$
g^{\lambda}=\frac{\hbar}{2 \pi k_{B} \mu_{0}} \frac{\Delta \rho_{0}}{T_{c 0} \lambda^{2}} \approx 0.24 \frac{\Delta \rho_{0}[\mu \Omega \mathrm{cm}]}{T_{c 0}[\mathrm{~K}]}
$$

where $\Delta \rho_{0}$ is the change in the residual resistivity due to irradiation and we use London penetration depth, $\lambda=200 \mathrm{~nm}$ [42]. This method provides a meaningful estimate of the single scattering rate related to directly measurable quantities [41]. We use Eq. (1) to plot the data in Fig. 3.

Discussion.-The residual resistivity change $\Delta \rho_{0}$ induced by irradiation is the most useful measure of the scattering since the rate $g$ itself is not directly measurable. To avoid the ambiguity in evaluating the generalized scattering rate [7], we use the same set of parameters to calculate $T_{c}$ and the residual resistivity in the Drude approximation [9]. As shown in Fig. 2, the increase of the resistivity induced by the electron irradiation is practically $T$ independent, meaning we can ignore the interference processes between inelastic and elastic scattering. We therefore calculate $\Delta \rho_{0}$ in the same $t$-matrix framework used in earlier studies (see, for example, Refs. [7,9]), assuming a two-band model. We further take all of the defects to be pointlike and scatter within a given band with the potential $v$ (intraband) and between the bands with the potential $u$ (interband). The ratio of inter- to intraband scattering, $\alpha \equiv u / v$, is a crucial parameter determining the rate of $T_{c}$ suppression [7,9].

Near $T_{c}$, the equations for the self-energies and superconducting gap can be linearized. To further simplify and avoid multiple free parameters, we take equal densities of states $N_{0}$ for both bands and the same intraband scattering strength $v$ and interband scattering $u$. Now we can write the following for $t_{c}=T_{c} / T_{c 0}$ :

$$
\log t_{c}=\Psi\left(\frac{1}{2}\right)-\Psi\left(\frac{1}{2}+\frac{2 g_{p}}{t_{c}}\right)
$$

Here, $g_{p}$ is the pair-breaking parameter, which reads

$$
g_{p}=\frac{1}{4 \pi \tau T_{c 0}} \frac{\alpha^{2}}{\left[1+\eta^{2}\left(1+\alpha^{2}\right)\right]^{2}-4 \alpha^{2} \eta^{2}},
$$


where $\tau^{-1}=2 n_{\text {imp }} \pi N_{0} v^{2}, \eta=\pi N_{0} v$, and $N_{0}$ is the total density of states. The unitary limit is achieved by taking $\eta \rightarrow \infty$.

The total dc conductivity in the $x$ direction is the sum of the Drude conductivities of the two bands, $\sigma=\sigma_{a}+\sigma_{b}$, with $\sigma_{\alpha}=2 e^{2} N_{\alpha}\left\langle v_{\alpha, x}^{2}\right\rangle \tau_{\alpha}$, where $v_{\alpha, x}$ is the component of the Fermi velocity in the $x$ direction and $\tau_{\alpha}$ is the corresponding single-particle relaxation time obtained from the self-energy in the $t$-matrix approximation, $\tau_{\alpha}^{-1}=-2 \operatorname{Im} \Sigma_{\alpha}^{(0)}$, containing contributions from both intraband and interband impurity scattering processes. The transport time is the same as the single-particle lifetime within this model because of our assumption of pointlike $s$-wave scatterers, meaning that the vertex corrections vanish in the limit where the Fermi surface pockets corresponding to the two bands are small.

Figure 4 shows the variation of $T_{c} / T_{c 0}$ with residual resistivity for three possible values of the interband and intraband potentials. Our aim here is not to fix these values-this is not possible since there are three independent parameters, $\tau^{-1}, u$, and $v$. However, we show that fits to the data can reproduce any experimental rate of the suppression of the $T_{c}$ by disorder. The experimental data

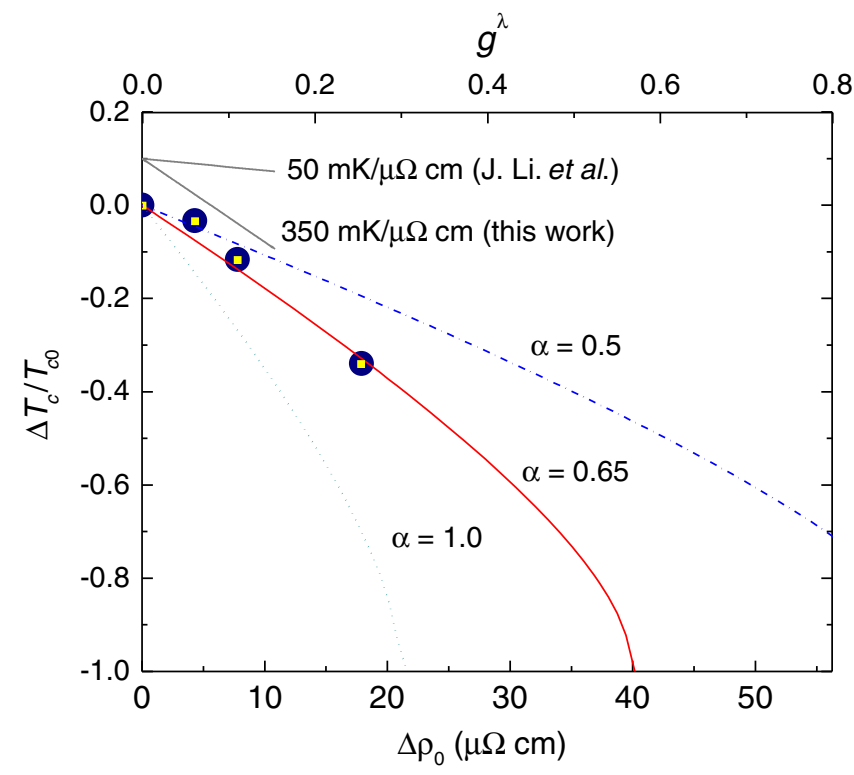

FIG. 4. Experimental data for $\Delta T_{c} / T_{c 0}$ (symbols) versus measured change in resistivity $\Delta \rho_{0}$ for single crystal of $\mathrm{Ba}\left(\mathrm{Fe}_{1-x} \mathrm{Ru}_{x}\right)_{2} \mathrm{As}_{2}(x=0.24)$. Lines are the fits to a $t$-matrix calculation for disorder self-energy with a fixed $\eta=0.74$, assuming the same density of states and taking Fermi velocity of $v_{F}=2 \times 10^{6} \mathrm{~m} / \mathrm{s}$ for both bands. Three theory lines correspond to the ratio of interband to intraband scattering $\alpha=1.0$, 0.65 , and 0.5 . Solid straight lines show average $T_{c}$ suppression of $50 \mathrm{mK} / \mu \Omega \mathrm{cm}$ by transition metal impurities $M$ substituted into $\mathrm{Ba}_{0.5} \mathrm{~K}_{0.5}\left(\mathrm{Fe}_{1-x} \mathrm{M}_{x}\right)_{2} \mathrm{As}_{2}$ from Ref. [11] and obtained in this work $350 \mathrm{mK} / \mu \Omega \mathrm{cm}$. The top axis shows dimensionless scattering rate $g^{\lambda}$ calculated from the resistivity and penetration depth, Eq. (1) shown by symbols agree quite reasonably with the theory. Note that we have excluded any possible anisotropy in order parameters so far, because determining order parameter anisotropy based only on $T_{c}$ suppression is not possible. In addition, the results reported in Ref. [9] explicitly showed that the gap anisotropy does not affect the qualitative features of the $T_{c}$ suppression problem for the $s_{ \pm}$state.

On the same plot, we show a line indicating the $T_{c}$ suppression found by Li et al. [11] for a variety of different transition metal impurities (including magnetic Mn ions), all of which suppress $T_{c}$ at roughly the same rate, a factor of 7 slower than that caused by the irradiation in our study. We note, however, that another isovalently substituted system, $\mathrm{BaFe}_{2}\left(\mathrm{As}_{1-x} \mathrm{P}_{x}\right)_{2}$, at optimal doping shows a remarkably similar rate of $340 \mathrm{mK} / \mu \Omega \mathrm{cm}$. We also note that the pure sample used by $\mathrm{Li}$ et al. had initial residual resistivity significantly higher than the unirradiated samples used in our study. Nevertheless, the remarkable difference between the $T_{c}$ suppression rates in the two cases is clear. We have now shown that there are types of defects produced by $e^{-}$irradiation, presumably Frenkel pairs of Fe vacancies and interstitials, that suppress $T_{c}$ at a rate much closer to the "ideal" Abrikosov-Gor'kov rate expected for the "symmetric model" $s_{ \pm}$state. Since such a fast $T_{c}$ suppression due to nonmagnetic disorder cannot take place for an $s_{++}$state, we rule out a non-sign-changing $s$-wave state at least for the 122 materials. This conclusion is also consistent with the similar pair-breaking rates for magnetic and nonmagnetic impurities in Ref. [11]. It disagrees, however, with a recent detailed five-orbital study of $T_{c}$ suppression by transition metal impurities [24] modeled by $a b$ initio calculations [43] that found that $T_{c}$ should be suppressed to zero at a critical resistivity of $\Delta \rho_{0} \sim 10 \mu \Omega \mathrm{cm}$, in contrast to the observed values of $\sim 1 \mathrm{~m} \Omega \mathrm{cm}$ in Ref. [11]. We cannot reconcile the claim of $s_{++}$pairing in Ref. [24] with the very small critical resistivity observed here.

One obvious concern is that this conclusion might be invalidated if electron irradiation creates magnetic defects, which would indeed act as strong pair breakers in an $s_{++}$ state. We examine the low-temperature penetration depth data on the irradiated samples in each case for signs of a low-temperature upturn that would indicate the contribution of magnetic defects to the superfluid density [44] and find no such terms. We are therefore confident that the evolution of the superconducting transition temperature $T_{c}$ with the electron irradiation-induced increase of residual resistivity in $\mathrm{Ba}\left(\mathrm{Fe}_{1-x} \mathrm{Ru}_{x}\right)_{2} \mathrm{As}_{2}$ is fully consistent with the generalized treatment of the impurity scattering within the $s_{ \pm}$pairing in iron-based superconductors. This conclusion is also supported by the electron irradiation study of a known two-band, but $s_{++}$superconductor, $\mathrm{MgB}_{2}$, where $T_{c}$ remains practically unchanged [38]. 


\section{ACKNOWLEDGMENTS}

We thank the SIRIUS team, B. Boizot, V. Metayer, and J. Losco, for help with the electron irradiation. We also thank C. van der Beek, A. Chubukov, R. Fernandes, H. Kontani, D. Maslov, I. Mazin, T. Shibauchi, and T. Tamegai for useful discussions. The work in Ames was supported by the U.S. Department of Energy (DOE), Office of Science, Basic Energy Sciences, Materials Science and Engineering Division. Ames Laboratory is operated for the U.S. DOE by Iowa State University under Contract No. DE-AC0207CH11358. Work at University of Florida was partially supported by DOE Grant No. DE-FG02-05ER46236. The work at the Ecole Polytechnique was supported by EMIR network, Proposal No. 11-11-0121. R. P. acknowledges the St. Gobain Chair position at the Ecole Polytechnique. V. M. acknowledges support from the Center for Emergent Superconductivity, an Energy Frontier Research Center funded by the U.S. DOE, Office of Science, under Award No. DE-AC0298CH1088.

[1] P. W. Anderson, Theory of Dirty Superconductors, J. Phys. Chem. Solids Suppl. 11, 26 (1959).

[2] A. A. Abrikosov and L. P. Gor'kov, Theory of Superconducting Alloys with Paramagnetic Impurities, Zh. Eksp. Teor. Fiz. 39, 1781 (1960) [Contribution to the Theory of Superconducting Alloys with Paramagnetic Impurities, Sov. Phys. JETP 12, 1243 (1961)].

[3] R. Balian and N. R. Werthamer, Superconductivity with Pairs in a Relative p Wave, Phys. Rev. 131, 1553 (1963).

[4] L. A. Openov, Critical Temperature of an Anisotropic Superconductor Containing Both Nonmagnetic and Magnetic Impurities, Phys. Rev. B 58, 9468 (1998).

[5] Y. Senga and H. Kontani, Impurity Effects in Sign-Reversing Fully Gapped Superconductors: Analysis of FeAs Superconductors, J. Phys. Soc. Jpn. 77, 113710 (2008).

[6] S. Onari and H. Kontani, Violation of Anderson's Theorem for the Sign-Reversing s-Wave State of Iron-Pnictide Superconductors, Phys. Rev. Lett. 103, 177001 (2009).

[7] D. V. Efremov, M. M. Korshunov, O. V. Dolgov, A. A. Golubov, and P. J. Hirschfeld, Disorder-Induced Transition between $s_{ \pm}$and $s_{++}$States in Two-Band Superconductors, Phys. Rev. B 84, 180512 (2011).

[8] R. M. Fernandes, M. G. Vavilov, and A. V. Chubukov, Enhancement of $t_{c}$ by Disorder in Underdoped Iron Pnictide Superconductors, Phys. Rev. B 85, 140512 (2012).

[9] Y. Wang, A. Kreisel, P. J. Hirschfeld, and V. Mishra, Using Controlled Disorder to Distinguish $s_{ \pm}$and $s_{++}$Gap Structure in Fe-Based Superconductors, Phys. Rev. B 87, 094504 (2013).

[10] P. J. Hirschfeld, M. M. Korshunov, and I. I. Mazin, Gap Symmetry and Structure of Fe-Based Superconductors, Rep. Prog. Phys. 74, 124508 (2011).

[11] J. Li, Y.F. Guo, S. B. Zhang, J. Yuan, Y. Tsujimoto, X. Wang, C. I. Sathish, Y. Sun, S. Yu, W. Yi, K. Yamaura, E. Takayama-Muromachiu, Y. Shirako, M. Akaogi, and
H. Kontani, Superconductivity Suppression of $\mathrm{Ba}_{0.5} \mathrm{~K}_{0.5} \mathrm{Fe}_{2-2 x} \mathrm{M}_{2 x} \mathrm{As}_{2}$ Single Crystals by Substitution of Transition Metal ( $M=\mathrm{Mn}, \mathrm{Ru}, \mathrm{Co}, \mathrm{Ni}, \mathrm{Cu}$, and $\mathrm{Zn}$ ), Phys. Rev. B 85, 214509 (2012).

[12] P. Cheng, B. Shen, J. Hu, and H. H. Wen, Contrasting Impurity Scattering and Pair-Breaking Effects by Doping $M n$ and $\mathrm{Zn}$ in $\mathrm{Ba}_{0.5} K_{0.5} \mathrm{Fe}_{2-2 x} \mathrm{~m}_{2 x} \mathrm{As}_{2}$, Phys. Rev. B 81, 174529 (2010).

[13] S. Konbu, K. Nakamura, H. Ikeda, and R. Arita, Effects of Transition-Metal Substitution in the Iron-Based Superconductor LaFeAsO: Momentum- and Real-Space Analysis from First Principles, Solid State Commun. 152, 728 (2012).

[14] J. Li, Y. Guo, S. Zhang, S. Yu, Y. Tsujimoto, H. Kontani, K. Yamaura, and E. Takayama-Muromachi, Linear Decrease of Critical Temperature with Increasing Zn Substitution in the Iron-Based Superconductor $\mathrm{BaFe}_{1.89-2 x} \mathrm{Zn}_{2 x} \mathrm{Co}_{0.11} \mathrm{As}_{2}$, Phys. Rev. B 84, 020513 (2011).

[15] A. C. Damask and G. J. Dienes, Point Defects in Metals (Gordon \& Breach Science Publishers Ltd, London, 1963).

[16] Y. Nakajima, Y. Tsuchiya, T. Taen, T. Tamegai, S. Okayasu, and M. Sasase, Enhancement of Critical Current Density in Co-Doped $\mathrm{BaFe}_{2} \mathrm{As}_{2}$ with Columnar Defects Introduced by Heavy-Ion Irradiation, Phys. Rev. B 80, 012510 (2009).

[17] H. Kim, R. T. Gordon, M. A. Tanatar, J. Hua, U. Welp, W. K. Kwok, N. Ni, S. L. Bud'ko, P. C. Canfield, A. B. Vorontsov, and R. Prozorov, London Penetration Depth in $\mathrm{Ba}\left(\mathrm{Fe}_{1-x} \mathrm{~T}_{x}\right)_{2} \mathrm{As}_{2}(T=C o, N i)$ Superconductors Irradiated with Heavy Ions, Phys. Rev. B 82, 060518 (2010).

[18] R. Prozorov, M. A. Tanatar, B. Roy, N. Ni, S. L. Bud'ko, P. C. Canfield, J. Hua, U. Welp, and W. K. Kwok, Magnetooptical Study of $\mathrm{Ba}\left(\mathrm{Fe}_{1-x} \mathrm{M}_{x}\right){ }_{2} \mathrm{As}_{2}(M=\mathrm{Co}$ and $\mathrm{Ni})$ Single Crystals Irradiated with Heavy Ions, Phys. Rev. B 81, 094509 (2010).

[19] J. Murphy, M. A. Tanatar, H. Kim, W. Kwok, U. Welp, D. Graf, J. S. Brooks, S. L. Bud'ko, P. C. Canfield, and R. Prozorov, Effect of Heavy-Ion Irradiation on London Penetration Depth in Overdoped $\mathrm{Ba}\left(\mathrm{Fe}_{1-x} \mathrm{Co}_{x}\right)_{2} \mathrm{As}_{2}$, Phys. Rev. B 88, 054514 (2013).

[20] L. Fang, Y. Jia, J. A. Schlueter, A. Kayani, Z. L. Xiao, H. Claus, U. Welp, A. E. Koshelev, G. W. Crabtree, and W. K. Kwok, Doping- and Irradiation-Controlled Pinning of Vortices in $\mathrm{BaFe}_{2}\left(\mathrm{As}_{1-x} \mathrm{P}_{x}\right)_{2}$ Single Crystals, Phys. Rev. B 84, 140504 (2011).

[21] Y. Nakajima, T. Taen, Y. Tsuchiya, T. Tamegai, H. Kitamura, and T. Murakami, Suppression of the Critical Temperature of Superconducting $\mathrm{Ba}\left(\mathrm{Fe}_{1-x} \mathrm{Co}_{x}\right)_{2} \mathrm{As}_{2}$ by Point Defects from Proton Irradiation, Phys. Rev. B 82, 220504 (2010).

[22] T. Taen, F. Ohtake, H. Akiyama, H. Inoue, Y. Sun, S. Pyon, T. Tamegai, and H. Kitamura, Pair-Breaking Effects Induced by $3 \mathrm{MeV}$ Proton Irradiation in $\mathrm{Ba}_{1-x} \mathrm{~K}_{x} \mathrm{Fe}_{2} \mathrm{As}_{2}$, Phys. Rev. B 88, 224514 (2013).

[23] C. Tarantini, M. Putti, A. Gurevich, Y. Shen, R. K. Singh, J. M. Rowell, N. Newman, D. C. Larbalestier, P. Cheng, Y. Jia, and H.H. Wen, Suppression of the Critical Temperature of Superconducting NdFeAs(OF) Single Crystals by Kondo-like Defect Sites Induced by $\alpha$-Particle Irradiation, Phys. Rev. Lett. 104, 087002 (2010).

[24] Y. Yamakawa, S. Onari, and H. Kontani, Effect of Realistic Finite-Size Impurities on $T_{c}$ in Fe-Based Superconductors 
Based on the Five-Orbital Tight-Binding Model, Phys. Rev. B 87, 195121 (2013).

[25] C. J. van der Beek, S. Demirdi, D. Colson, F. Rullier-Albenque, Y. Fasano, T. Shibauchi, Y. Matsuda, S. Kasahara, P. Gierlowski, and M. Marcin Konczykowski, Electron Irradiation of $\mathrm{Co}, \mathrm{Ni}$, and $\mathrm{P}$-doped $\mathrm{BaFe}_{2} \mathrm{As}_{2}$-Type Iron-Based Superconductors, J. Phys. Conf. Ser. 449, 012023 (2013).

[26] F. Rullier-Albenque, D. Colson, A. Forget, P. Thuery, and S. Poissonnet, Hole and Electron Contributions to the Transport Properties of $\mathrm{Ba}\left(\mathrm{Fe}_{1-x} \mathrm{Ru}_{x}\right)_{2} \mathrm{As}_{2}$ Single Crystals, Phys. Rev. B 81, 224503 (2010).

[27] A. Thaler, N. Ni, A. Kracher, J. Q. Yan, S. L. Bud'ko, and P.C. Canfield, Physical and Magnetic Properties of $\mathrm{Ba}\left(\mathrm{Fe}_{1-x} \mathrm{Ru}_{x}\right)_{2} \mathrm{As}_{2}$ Single Crystals, Phys. Rev. B 82, 014534 (2010).

[28] H. Shishido, A. F. Bangura, A. I. Coldea, S. Tonegawa, K. Hashimoto, S. Kasahara, P. M. C. Rourke, H. Ikeda, T. Terashima, R. Settai, Y. Onuki, D. Vignolles, C. Proust, B. Vignolle, A. McCollam, Y. Matsuda, T. Shibauchi, and A. Carrington, Evolution of the Fermi Surface of $\mathrm{BaFe}_{2}\left(\mathrm{As}_{1-x} \mathrm{P}_{x}\right)_{2}$ on Entering the Superconducting Dome, Phys. Rev. Lett. 104, 057008 (2010).

[29] R. S. Dhaka, C. Liu, R. M. Fernandes, R. Jiang, C. P. Strehlow, T. Kondo, A. Thaler, J. Schmalian, S. L. Bud'ko, P. C. Canfield, and A. Kaminski, What Controls the Phase Diagram and Superconductivity in Ru-Substituted $\mathrm{BaFe}_{2} \mathrm{As}_{2}$ ?, Phys. Rev. Lett. 107, 267002 (2011).

[30] N. Xu, T. Qian, P. Richard, Y. B. Shi, X. P. Wang, P. Zhang, Y. B. Huang, Y. M. Xu, H. Miao, G. Xu, G. F. Xuan, W. H. Jiao, Z. A. Xu, G. H. Cao, and H. Ding, Effects of Ru Substitution on Electron Correlations and Fermi-Surface Dimensionality in $\mathrm{Ba}\left(\mathrm{Fe}_{1-x} \mathrm{Ru}_{x}\right)_{2} \mathrm{As}_{2}$, Phys. Rev. B 86, 064505 (2012).

[31] L. Wang, T. Berlijn, Y. Wang, C.-H. Lin, P. J. Hirschfeld, and Wei Ku, Effects of Disordered Ru Substitution in $\mathrm{BaFe}_{2} \mathrm{As}_{2}$ : Possible Realization of Superdiffusion in Real Materials, Phys. Rev. Lett. 110, 037001 (2013).

[32] K. Hashimoto, K. Cho, T. Shibauchi, S. Kasahara, Y. Mizukami, R. Katsumata, Y. Tsuruhara, T. Terashima, H. Ikeda, M. A. Tanatar, H. Kitano, N. Salovich, R. W. Giannetta, P. Walmsley, A. Carrington, R. Prozorov, and Y. Matsuda, A Sharp Peak of the Zero-Temperature Penetration Depth at Optimal Composition in $\mathrm{BaFe}_{2}\left(\mathrm{As}_{1-x} \mathrm{Px}\right)_{2}$, Science 336, 1554 (2012).
[33] A. A. Golubov and I. I. Mazin, Effect of Magnetic and Nonmagnetic Impurities on Highly Anisotropic Superconductivity, Phys. Rev. B 55, 15146 (1997).

[34] M. Putti, R. Vaglio, and J. M. Rowell, Radiation Effects on $\mathrm{MgB}_{2}$ : A Review and a Comparison with A15 Superconductors, Supercond. Sci. Technol. 21, 043001 (2008).

[35] R. H. T. Wilke, S. L. Bud'ko, P. C. Canfield, J. Farmer, and S. T. Hannahs, Systematic Study of the Superconducting and Normal-State Properties of Neutron-Irradiated $\mathrm{MgB}_{2}$, Phys. Rev. B 73, 134512 (2006).

[36] M. Putti, P. Brotto, M. Monni, E. Galleani d'Agliano, A. Sanna, and S. Massidda, Intraband vs. Interband Scattering Rate Effects in Neutron Irradiated $\mathrm{MgB}_{2}$, Europhys. Lett. 77, 57005 (2007).

[37] A. A. Blinkin, V. V. Derevyanko, A. N. Dovbnya, T. V. Sukhareva, V. A. Finkel', and I. N. Shlyakhov, Effect of Electron Irradiation on the Structure and Properties of the $\mathrm{MgB}_{2}$ Superconductor, Phys. Solid State 48, 2037 (2006).

[38] T. Klein, R. Marlaud, C. Marcenat, H. Cercellier, M. Konczykowski, C. J. van der Beek, V. Mosser, H. S. Lee, and S. I. Lee, First-Order Transition in the Magnetic Vortex Matter in Superconducting $\mathrm{MgB}_{2}$ Tuned by Disorder, Phys. Rev. Lett. 105, 047001 (2010).

[39] M. A. Tanatar, N. Ni, S. L. Bud'ko, P. C. Canfield, and R. Prozorov, Field-Dependent Transport Critical Current in Single Crystals of $\mathrm{Ba}\left(\mathrm{Fe}_{1-x} T M_{x}\right)_{2} \mathrm{As}_{2} \quad(T M=\mathrm{Co}, \mathrm{Ni})$ Superconductors, Supercond. Sci. Technol. 23, 054002 (2010).

[40] S. Onari and H. Kontani, Nonmagnetic Impurity Effects and Neutron Scattering Spectrum in Iron Pnictides, Physica C (Amsterdam) 470C, 1007 (2010).

[41] V. G. Kogan, Pair Breaking in Iron Pnictides, Phys. Rev. B 80, 214532 (2009).

[42] R. T. Gordon, H. Kim, M. A. Tanatar, R. Prozorov, and V. G. Kogan, London Penetration Depth and Strong Pair Breaking in Iron-Based Superconductors, Phys. Rev. B 81, 180501 (2010).

[43] K. Nakamura, R. Arita, and H. Ikeda, First-Principles Calculation of Transition-Metal Impurities in LaFeAsO, Phys. Rev. B 83, 144512 (2011).

[44] J. R. Cooper, Power-Law Dependence of the ab-Plane Penetration Depth in $\mathrm{Nd}_{1.85} \mathrm{Ce}_{0.15} \mathrm{CuO}_{4-y}$, Phys. Rev. B 54, R3753 (1996). 